\title{
Two infinite families of nonadditive quantum error-correcting codes
}

\author{
Sixia $\mathrm{Yu}^{1,2}$, Qing Chen ${ }^{1,2}$, and C. H. $\mathrm{Oh}^{2}$ \\ ${ }^{1}$ Hefei National Laboratory for Physical Sciences at Microscale and Department of Modern Physics \& \\ Department of Modern Physics University of Science and Technology of China, Hefei 230026, P.R. China \\ ${ }^{2}$ Physics Department, National University of Singapore, 2 Science Drive 3, Singapore 117542
}

\begin{abstract}
We construct explicitly two infinite families of genuine nonadditive 1-error correcting quantum codes and prove that their coding subspaces are 50\% larger than those of the optimal stabilizer codes of the same parameters via the linear programming bound. All these nonadditive codes can be characterized by a stabilizer-like structure and thus their encoding circuits can be designed in a straightforward manner.
\end{abstract}

One major family of quantum error-correcting codes (QECCs) [1, 2, 3, 4], which are powerful tools to fight the quantum noises in various quantum informational processes, are called as additive or stabilizer codes [5, 6, 7, 8]. The coding subspace of a stabilizer code is specified by the joint +1 eigenspace a group of commuting multilocal (direct product of) Pauli operators. Usually $[[n, k, d]]$ denotes a stabilizer code of length $n$, the number of physical qubits, and distance $d$, i.e., correcting up to $\left\lfloor\frac{d-1}{2}\right\rfloor$-qubit errors, that encodes $k \log$ ical qubits $\left(2^{k}\right.$-dimensional subspace).

The first example of nonadditive codes, codes that cannot be described within the framework of stabilizer, was an infinite family of 1-error-detecting codes [9, 10], e.g., $((5,6,2))$, with coding subspaces being 50\% larger than the optimal stabilizer codes of the same parameters. Recently another family of 1-error detecting codes with still larger encoding subspaces has been constructed in [11] and slightly improved in [12]. Here we have denoted by $((n, K, d))$ a nonadditive code of length $n$ and distance $d$ that encodes a $K$-dimensional logical subspace (about $\log _{2} K$ logical qubits).

The first example of nonadditive code [13], namely $((9,12,3))$, that outperforms all the stabilizer codes of the same length while capable of correcting arbitrary single qubit errors has recently been constructed via a graphical approach based on graph states. Later on an optimal 10-qubit code $((10,24,3))$ has been found via a comprehensive computer search [12]. Recently a family of codes of distance 8 that encode 3 more logical qubits than the best known stabilizer codes have been constructed based on nonlinear classical codes [14]. However the possibility of being equivalent to some subcode of an optimal stabilizer code or even a stabilizer code of the same parameters under local unitary transformations has not yet been excluded.

Generally, being without a stabilizer structure, the nonadditive codes promise larger coding subspaces while they are harder to construct and identify than the stabilizer codes. On one hand there is no systematic construction so far and all the good codes are found via computer search [12, 15], which is impossible for a relatively large length (e.g. $n \geq 11$ ). On the other hand an obvious criterion for a genuine nonadditive code is to check whether or not its coding subspace is larger than all the stabilizer codes of the same parameters. However the exact bound for stabilizer codes is generally unknown. As a result it is of interest to find nonadditive error-correcting code when the length tends to infinity that outperforms all the stabilizer codes of the same parameters.

In this Letter we shall construct two infinite families of genuine nonadditive 1-error-correcting codes with coding subspaces being 50\% larger then the corresponding optimal 1error-correcting stabilizer codes of the same parameters to show that the nonadditive error-correcting codes outperform the stabilizer codes even when the length $n$ tends to infinity. All the nonadditive codes are characterized by a stabilizer-like structure and therefore the encoding-decoding circuits can be designed in a straightforward manner.

Two families of nonadditive 1-error correcting codes that we shall construct have the following parameters

$$
\mathbb{D}_{(m, a)}=\left(\left(N_{m}^{a}, \frac{3}{2} 2^{N_{m}^{a}-2 m-6}, 3\right)\right)
$$

where $N_{m}^{a}=\frac{2^{2 m+5}-5}{3}+a$ with $a=0,1$ and $m \geq 1$. To ensure that they are genuine nonadditive we shall prove that the corresponding optimal 1-error-correcting stabilizer codes of the same length have parameters

$$
\left[\left[N_{m}^{a}, N_{m}^{a}-2 m-6,3\right]\right]
$$

by working out analytically the linear programming bound for the lengths $N_{m}^{a}$. Notice that the quantum Hamming bound permits exactly one more logical qubit, i.e., $\left\lceil\log _{2}\left(3 N_{m}^{a}+\right.\right.$ $1)\rceil=2 m+5$. The first nonadditive codes of these two families are $\left(\left(41,3 \cdot 2^{32}, 3\right)\right)$ and $\left(\left(42,3 \cdot 2^{33}, 3\right)\right)$ respectively while the optimal stabilizer codes have the parameters $[[41,33,3]]$ and $[[42,34,3]]$.

Our construction is based on a family of stabilizer codes of lengths $\left\{2^{2 r+3}\right\}_{r=1}^{m}[5,8]$ and two nonadditive codes of length 9 and 10 discovered recently [12, 13] and is a kind of pasting stabilizer codes with nonadditive codes that generalizes the pasting of stabilizer codes in Ref.[16]. We denote by $\mathcal{X}_{v}, \mathcal{Z}_{v}$ three Pauli operators acting nontrivially only on some qubit labeled by $v$ and by $\mathcal{I}$ the identity operator. Furthermore for a given index set $U$ we denote $\mathcal{X}_{U}=\prod_{v \in U} \mathcal{X}$ and similarly for other Pauli operators.

Let us look at the optimal stabilizer of length $2^{2 r+3}$ at first. According to Ref.[5] the stabilizer of the code has $2 r+5$ generators with two of them being $\mathcal{X}_{U_{r}}$ and $\mathcal{Z}_{U_{r}}$ where we have labeled $2^{2 r+3}$ physical qubits with $U_{r}=\left\{1,2,3, \ldots, 2^{2 r+3}\right\}$. The remaining $2 r+3$ generators are given by

$$
\left\{\mathcal{S}_{k}^{r}=\mathcal{X}^{\mathbf{h}_{k}} \mathcal{Z}^{\mathbf{h}_{k-1}+\mathbf{h}_{1}+\mathbf{h}_{2 r+3}} \mid k \in U_{r}\right\} .
$$


Here $\mathbf{h}_{k}$ denotes a $2^{2 r+3}$-dim vector that is the $k$-th row of a $(2 r+3) \times 2^{2 r+3}$ matrix $H_{r}=\left[c_{0}, c_{1}, \ldots, c_{2^{2 r+3}-1}\right]$ whose $k$-th column $c_{k}$ being the binary representation of $k$, e.g., $c_{1}^{T}=(0,0, \ldots, 1)$ and $c_{2^{2 r+3}-1}=(1,1, \ldots, 1)$ and $\mathbf{h}_{0}=\mathbf{0}$ is the zero vector. And for a vector $\mathbf{h}$ with components $\left\{h_{v} \mid v \in U_{r}\right\}$ we have denoted $\mathcal{X}^{\mathbf{h}}=\prod_{v \in U_{r}} \mathcal{X}_{v}^{h_{v}}$ and $\mathcal{Z}^{\mathbf{h}}=\prod_{v \in U_{r}} \mathcal{Z}_{v}^{h_{v}}$.

Despite their nonadditiveness the codes $((9,12,3))$ and $(10,24,3))$ admit a stabilizer-like structure and can be most conveniently formulated by using the graph states [20, 21]. We denote by $G=(V, E)$ a simple undirected graph with a set $V$ of vertices and a set $E$ of edges. Two vertices are connected with an edge iff $\{a, b\} \in E$. Two graphs $G_{a}(a=0,1)$ on $\left|V_{0}\right|=9$ and $\left|V_{1}\right|=10$ vertices are shown in Fig.1. By labeling $|V|$ qubits by $V$ we can define the graph state corresponding to the graph $G$ a $|G\rangle=\mathcal{U}_{G}|+\rangle_{x}^{V}$ where

$$
\mathcal{U}_{G}=\prod_{\{a, b\} \in E} \frac{1+\mathcal{Z}_{a}+\mathcal{Z}_{b}-\mathcal{Z}_{a} \mathcal{Z}_{b}}{2},
$$

and $|+\rangle_{x}^{V}$ denotes the joint +1 eigenstate of $\mathcal{X}_{v}$ for $v \in V$. Obviously $\mathcal{U}_{G}^{2}=1$ and the graph state $|G\rangle$ is also the +1 joint eigenstate of the following $n$ stabilizers $\mathcal{G}_{v}=\mathcal{U}_{G} \mathcal{X}_{v} \mathcal{U}_{G}$.

From the graph state $|G\rangle$ a basis for the whole system a basis of the whole system can built $\left\{\mathcal{Z}_{C}|G\rangle \mid C \subseteq V\right\}$. For trivial graph with no edge the graph-state basis reads $\mathcal{Z}_{C}|+\rangle_{x}^{V}$. Any given collection of vertex subsets of $V$ will define a basis that spans a subspace which is referred to as the graph-state basis. With a graph and a collection of vertex subsets will define a subspace.

For the nonadditive code $((9,12,3)) \equiv \mathbb{D}_{(0,0)}$ we consider the loop graph $G_{0}=\left(V_{0}, E_{0}\right)$ on 9 vertices as shown in Fig.1a and corresponding graph state $\left|G_{0}\right\rangle$. The joint +1 eigenspace of the following 6 observables

$$
\begin{aligned}
\alpha_{1} & =\mathcal{U}_{G_{0}} \mathcal{X}_{\{3,8\}} \mathcal{U}_{G_{0}}, \\
\alpha_{2} & =\mathcal{U}_{G_{0}} \mathcal{X}_{\{6,2\}} \mathcal{U}_{G_{0}}, \\
\alpha_{3} & =\mathcal{U}_{G_{0}} \mathcal{X}_{\{9,5\}} \mathcal{U}_{G_{0}}, \\
\mathcal{A}_{1} & =\mathcal{U}_{G_{0}}\left(\mathcal{X}_{\{4,7,3,6,9\}} \mathcal{V}_{69}\right) \mathcal{U}_{G_{0}}, \\
\mathcal{A}_{2} & =\mathcal{U}_{G_{0}}\left(\mathcal{X}_{\{1,7,3,6\}} \mathcal{V}_{39}\right) \mathcal{U}_{G_{0}}, \\
\mathcal{A}_{3} & =\mathcal{U}_{G_{0}}\left(\mathcal{X}_{\{1,4,3,9\}} \mathcal{V}_{36}\right) \mathcal{U}_{G_{0}},
\end{aligned}
$$

is exactly the 12 -dim coding subspace of the code $\mathbb{D}_{(0,0)}$. Here
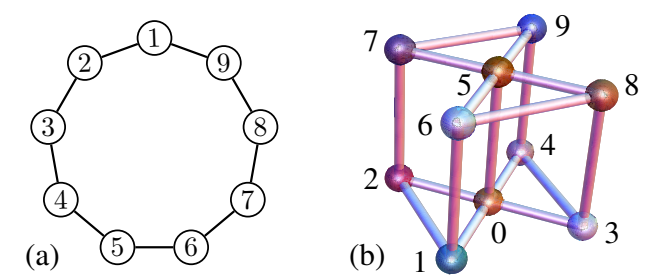

FIG. 1: Two graphs $G_{a}=\left(V_{a}, E_{a}\right)$ are shown with $V_{0}=$ $\{1,2, \ldots, 9\}$ and $V_{1}=\{0,1, \ldots, 9\}$. They provide the graph-state bases for two nonadditive codes $((9,12,3))$ and $((10,24,3))$. we have denoted $\mathcal{V}_{a b}=\frac{1}{2}\left(1+\mathcal{X}_{a}+\mathcal{X}_{b}-\mathcal{X}_{a} \mathcal{X}_{b}\right)$. If we denote $\mathcal{A}_{0}=\mathcal{A}_{1} \mathcal{A}_{2} \mathcal{A}_{3}$ we have $\operatorname{Tr}_{V_{0}} \mathcal{A}_{0}=2^{8}$. It should be noted that the code is nondegenerate, which can be easily seen from its weight distributions.

The nonadditive optimal code $((10,24,3)) \equiv \mathbb{D}_{(0,1)}$ has a graph-state basis corresponding to the graph $G_{1}=\left(V_{1}, E_{1}\right)$ on 10 vertices as shown in Fig.1b and the corresponding graph state is $\left|G_{1}\right\rangle$. Obviously the graph is unchanged under the following two permutations

$$
\pi=(14)(23)(69)(78), \quad \tau=(12)(34)(67)(89) .
$$

that act naturally on $V$. Two swapping operators $\mathcal{M}_{\pi}$ and $\mathcal{M}_{\tau}$ are defined via, e.g., $\mathcal{M}_{\pi} \mathcal{Z}_{C}|+\rangle_{x}^{V_{1}}=\mathcal{Z}_{\pi(C)}|+\rangle_{x}^{V_{1}}$ for an arbitrary $C \subseteq V$. For later use we define two controlled-swap operations with qubits 0 and 5 as sources respectively as

$$
\begin{aligned}
& \mathcal{T}_{\pi}=\frac{1}{2}\left(1+\mathcal{X}_{0}+\left(1-\mathcal{X}_{0}\right) \mathcal{M}_{\pi}\right), \\
& \mathcal{T}_{\tau}=\frac{1}{2}\left(1+\mathcal{X}_{5}+\left(1-\mathcal{X}_{5}\right) \mathcal{M}_{\tau}\right),
\end{aligned}
$$

which can be readily implemented via Toffli and control-not gates. According to Ref.[12], those 24 graph-state bases of the code $\mathbb{D}_{(0,1)}$ are given by $\left\{\left|C_{\mu \nu}^{i}\right\rangle=\mathcal{Z}_{C_{\mu \nu}^{i}}\left|G_{1}\right\rangle\right\}$ with $i=$ $1,2, \ldots, 6$ and $\mu, \nu=0,1$ where 24 subsets of $V_{1}$ are

$$
C_{\mu \nu}^{i}=\pi^{\mu} \circ \tau^{\nu}\left(C_{i}\right) \triangle \nu B \triangle \mu \tau^{\nu}(A)
$$

with $A \triangle B=A \cup B-A \cap B$ being the symmetric difference between two sets $A$ and $B$ and

$$
\begin{gathered}
A=\{0,2,3\}, B=\{5,1,2\}, C_{1}=\emptyset, \\
C_{2}=\{1,2,3,9\}, C_{3}=\{1,2,7,8\}, C_{4}=\{1,2,6,7,9\}, \\
C_{5}=\{1,3,7,8,9\}, C_{6}=\{1,3,4,6,7,9\} .
\end{gathered}
$$

It turns out that the nonadditive code $\mathbb{D}_{(0,1)}$ admits also a stabilizer-like structure. With the help of the following encoding operator

$$
\mathcal{U}_{\text {enc }}=\mathcal{Z}_{2} \mathcal{U}_{G_{1}} \mathcal{T}_{\tau} \mathcal{T}_{\pi} \mathcal{Z}_{2}
$$

it can be readily checked that the following 6 mutually commuting observables

$$
\begin{aligned}
& \beta_{1}=\mathcal{U}_{\text {enc }} \mathcal{X}_{\{2,3,7\}} \mathcal{U}_{\text {enc }}^{\dagger}, \\
& \beta_{2}=\mathcal{U}_{\text {enc }} \mathcal{X}_{\{6,7,8\}} \mathcal{U}_{\text {enc }}^{\dagger}, \\
& \beta_{3}=\mathcal{U}_{\text {enc }} \mathcal{X}_{\{3,4,6,9\}} \mathcal{U}_{\text {enc }}^{\dagger}, \\
& \mathcal{B}_{0}=\mathcal{U}_{\text {enc }}\left(\mathcal{X}_{6} \mathcal{V}_{67}\right) \mathcal{U}_{\text {enc }}^{\dagger}, \\
& \mathcal{B}_{1}=\mathcal{U}_{\text {enc }}\left(\mathcal{X}_{\{1,2\}} \mathcal{V}_{37}\right) \mathcal{U}_{\text {enc }}^{\dagger}, \\
& \mathcal{B}_{2}=\mathcal{U}_{\text {enc }}\left(\mathcal{X}_{4} \mathcal{V}_{36}\right) \mathcal{U}_{\text {enc }}^{\dagger}
\end{aligned}
$$

stabilize the coding subspace of $\mathbb{D}_{(0,1)}$, i.e., one one hand $\beta_{k}\left|C_{\mu \nu}^{i}\right\rangle=\mathcal{B}_{l}\left|C_{\mu \nu}^{i}\right\rangle=\left|C_{\mu \nu}^{i}\right\rangle$ for all possible $k, l, i, \mu, \nu$ and on the other hand the joint +1 eigenspace of these 6 observables, whose projector is given by

$$
\mathcal{P}_{1}=\prod_{i=1}^{3} \frac{1+\beta_{i}}{2} \prod_{i=0}^{2} \frac{1+\mathcal{B}_{i}}{2},
$$


TABLE I: The stabilizing observables of the nonadditive codes $\mathbb{D}_{m}^{a}$ whose physical qubits are labeled with $U_{m} \cup \ldots \cup U_{1} \cup V_{a}(a=0,1)$. The blank entries stand for suitable identity operators $\mathcal{I}_{U_{k}}$ or $\mathcal{I}_{V_{a}}$.

\begin{tabular}{c|ccllll}
\hline \hline & $U_{m}$ & $U_{m-1}$ & $\cdots$ & $U_{2}$ & $U_{1}$ & $V_{0}$ or $V_{1}$ \\
\hline $\mathcal{O}_{1}^{(0,1)}$ & $\mathcal{X}_{U_{m}}$ & & & & & \\
$\mathcal{O}_{2}^{(0,1)}$ & $\mathcal{Z}_{U_{m}}$ & & & & & \\
\hline $\mathcal{O}_{3}^{(0,1)}$ & $\mathcal{S}_{1}^{m}$ & $\mathcal{X}_{U_{m-1}}$ & & & & \\
$\mathcal{O}_{4}^{(0,1)}$ & $\mathcal{S}_{2}^{m}$ & $\mathcal{Z}_{U_{m-1}}$ & & & & \\
\hline $\mathcal{O}_{5}^{(0,1)}$ & $\mathcal{S}_{3}^{m}$ & $\mathcal{S}_{1}^{m-1}$ & & & & \\
$\vdots$ & $\vdots$ & $\vdots$ & $\ddots$ & & & \\
$\mathcal{O}_{2 m-4}^{(0,1)}$ & $\mathcal{S}_{2 m-6}^{m}$ & $\mathcal{S}_{2 m-8}^{m-1}$ & $\cdots$ & & & \\
\hline $\mathcal{O}_{2 m-3}^{(0,1)}$ & $\mathcal{S}_{2 m-5}^{m}$ & $\mathcal{S}_{2 m-7}^{m-1}$ & $\cdots$ & $\mathcal{X}_{U_{2}}$ & & \\
$\mathcal{O}_{2 m-2}^{(0,1)}$ & $\mathcal{S}_{2 m-4}^{m}$ & $\mathcal{S}_{2 m-6}^{m-1}$ & $\cdots$ & $\mathcal{Z}_{U_{2}}$ & & \\
\hline $\mathcal{O}_{2 m-1}^{(0,1)}$ & $\mathcal{S}_{2 m-3}^{m}$ & $\mathcal{S}_{2 m-5}^{m-1}$ & $\cdots$ & $\mathcal{S}_{1}^{2}$ & $\mathcal{X}_{U_{1}}$ & \\
$\mathcal{O}_{2 m}^{(0,1)}$ & $\mathcal{S}_{2 m-2}^{m}$ & $\mathcal{S}_{2 m-1}^{m-1}$ & $\cdots$ & $\mathcal{S}_{2}^{2}$ & $\mathcal{Z}_{U_{1}}$ & \\
\hline $\mathcal{O}_{2 m+1}^{(0,1)}$ & $\mathcal{S}_{2 m-1}^{m}$ & $\mathcal{S}_{2 m-1}^{m-1}$ & $\cdots$ & $\mathcal{S}_{3}^{2}$ & $\mathcal{S}_{1}^{1}$ & $\alpha_{1}$ or $\beta_{1}$ \\
$\mathcal{O}_{2 m+2}^{(0,1)}$ & $\mathcal{S}_{2 m}^{m}$ & $\mathcal{S}_{2 m-1}^{m-1}$ & $\cdots$ & $\mathcal{S}_{4}^{2}$ & $\mathcal{S}_{2}^{1}$ & $\alpha_{2}$ or $\beta_{2}$ \\
$\mathcal{O}_{2 m+1}^{(0,1)}$ & $\mathcal{S}_{2 m+1}^{m}$ & $\mathcal{S}_{2 m-1}^{m-1}$ & $\cdots$ & $\mathcal{S}_{5}^{2}$ & $\mathcal{S}_{3}^{1}$ & $\alpha_{3}$ or $\beta_{3}$ \\
$\mathcal{O}_{2 m+4}^{(0,1)}$ & $\mathcal{S}_{2 m+2}^{m}$ & $\mathcal{S}_{2 m}^{m-1}$ & $\cdots$ & $\mathcal{S}_{6}^{2}$ & $\mathcal{S}_{4}^{1}$ & $\mathcal{A}_{1}$ or $\mathcal{B}_{1}$ \\
$\mathcal{O}_{2 m+5}^{(0,1)}$ & $\mathcal{S}_{2 m+3}^{m}$ & $\mathcal{S}_{2 m+1}^{m-1}$ & $\cdots$ & $\mathcal{S}_{7}^{2}$ & $\mathcal{S}_{5}^{1}$ & $\mathcal{A}_{2}$ or $\mathcal{B}_{2}$ \\
\hline $\mathcal{O}_{2 m+6}^{(0,1)}$ & & & & & & $\mathcal{A}_{0}$ or $\mathcal{B}_{0}$ \\
\hline \hline
\end{tabular}

has exactly dimension 24 , i.e., $\operatorname{Tr}_{V_{1}} \mathcal{P}=24$ since $\operatorname{Tr}_{V_{1}} \mathcal{B}_{0}=$ $2^{9}$. An encoding circuit can therefore be designed in a similar manner as that of $((9,12,3))$ [13]. We note also that this nonadditive code is non-degenerate.

Now we are ready to present our construction. We consider $N_{m}^{a}$ qubits and label them by disjoint set $U_{m} \cup U_{m-1} \cup \ldots \cup$ $U_{1} \cup V_{a}$ with $\left|U_{k}\right|=2^{2 k+3}$ and $\left|V_{a}\right|=9+a$ with $k \leq m$ and $a=0,1$. We claim that the joint +1 eigenspace of those $2 m+$ 6 observables $\left\{\mathcal{O}_{i}^{(a)}\right\}_{i=1}^{2 m+6}$ with $a=0$ or 1 as defined in Table I is the code $\mathbb{D}_{(m, a)}$ in Eq.(1) with the following projector onto the coding subspace

$$
\mathcal{P}_{m}^{a}=\prod_{i=1}^{2 m+6} \frac{1+\mathcal{O}_{i}^{(a)}}{2} .
$$

In Table I observables $\mathcal{S}_{i}^{r}$ are defined in Eq. 3$\}$ and $\left\{\alpha_{i}, \mathcal{A}_{j}\right\}$, $\left\{\beta_{i}, \mathcal{B}_{j}\right\}$ are defined via Eq. (5) and Eq.(11) respectively. Blank entries represent suitable identity operators. By juxtaposition of some operators in the same row we mean their direct product.

First of all, these $2 m+6$ stabilizing observables detect all 2-qubit errors because firstly all errors happened on $U$-blocks or $V$ blocks can be detected because all the subcodes are pure 1-error correcting codes and secondly all two errors happened on different qubit blocks can always be detected by the stabilizer containing $\mathcal{X}_{U_{k}}$ and $\mathcal{Z}_{U_{k}}$ for some $k$. Thus we obtain a pure 1-error correcting codes of length $N_{m}^{a}$. Secondly, by noticing $\operatorname{Tr} \mathcal{O}_{i}^{(a)}=0$ for $i \leq 2 m+5$ and $\operatorname{Tr} \mathcal{O}_{2 m+6}^{(a)}=2^{N_{m}^{a}-1}$ we have

$$
\operatorname{Tr} \mathcal{P}_{m}^{a}=\frac{\operatorname{Tr}\left(1+\mathcal{O}_{2 m+6}^{(a)}\right)}{2^{2 m+6}}=\frac{3}{2} 2^{N_{m}^{a}-2 m-6} .
$$

Thus we obtain the 1-error correcting code of parameters exactly as given in Eq. (11). Now we shall demonstrate that its coding subspace is $50 \%$ larger than the corresponding optimal stabilizer codes so that our codes are genuine nonadditive codes that are neither equivalent to some stabilizer codes under local unitary transformations nor subcodes of some larger 1-error correcting stabilizer codes of the same length.

The quantum Hamming bound for a 1-error correcting stabilizer code, e.g., $n-k \geq\left\lceil\log _{2}(3 n+1)\right\rceil$ for a stabilizer code $[[n, k, 3]]$, being introduced initially for the non-degenerate codes, is valid for both degenerate and generate codes of distance 3 and 5 [6] and of a large enough length [19]. In the case of $n=N_{m}^{a}$ we have the quantum Hamming bound $n-k \geq 2 m+5$. This is not enough to prove the nonadditiveness of our codes. However by working out analytically the linear programming bound we have

Theorem If there exists a stabilizer code $\left[\left[N_{m}^{a}, k, 3\right]\right]$, degenerate or non-degenerate, with $N_{m}^{a}=\frac{2^{2 m+5}-5}{3}+a$ and $m \geq 0$ and $a=0,1$ then $N_{m}^{a}-k \geq 2 m+6$.

Proof. Given a stabilizer code $[[n, k, d]]$ its weight distributions $\left\{A_{i}\right\}_{i=0}^{n}$ is defined by

$$
A_{i}=\frac{1}{2^{2 k}} \sum_{|\omega|=i}\left|\operatorname{Tr}\left(P \mathcal{E}_{\omega}\right)\right|^{2} \quad(i=0,1, \ldots, n),
$$

where the summation is over all errors supported on $i$ qubits and $P$ is the projector onto the coding subspace. It is obvious that $A_{i} \geq 0, A_{0}=1$, and $\sum_{i} A_{i}=2^{s}$ so that $\left\{A_{i} / 2^{s}\right\}$ can be regarded as a probability distribution with $s=n-k$. For an arbitrary function $f(x)$ we denote its average

$$
\langle f(x)\rangle \equiv \frac{1}{2^{s}} \sum_{i=0}^{d} f(i) A_{i} .
$$

In the following we shall formulate a subset of the linear programming bound for 1-error correcting code, which serves our purpose perfectly. For a complete set of linear programming bound see Ref.[8, 17].

Linear Programming bound (Restricted) If there exists a stabilizer code $[[n, k, 3]]$ then the following conditions hold true

$$
\begin{array}{r}
A_{1}=\langle 3 n-4 x\rangle, \\
A_{2}=\frac{1}{2}\left\langle(4 x-3 n+1)^{2}-3 n-1\right\rangle, \\
\sum_{i=0}^{\left\lfloor\frac{n}{2}\right\rfloor} A_{2 i} \geq 2^{s-1} .
\end{array}
$$

In the case of $a=0$, i.e., $n=N_{m}^{0}$ with $m \geq 0$ we introduce a nonnegative function $f(x)=(3 n+1-4 x)^{2}$ and it is easy 
to check that as long as $n \geq 5$

$$
\begin{aligned}
& f(0)=(3 n+1)^{2}>(3 n+5)(3 n-7)+16, \\
& f(1)=(3 n-3)^{2}>4(3 n+5), \\
& f(2)=(3 n-7)^{2}>2(3 n+5)+16 .
\end{aligned}
$$

If there exists a stabilizer code $[[n, k, 3]]$ then Eqs. $17 \mathrm{a} \mid 17 \mathrm{c}$ must hold. As a result

$$
\begin{gathered}
\langle f(x)\rangle=3 n+1+4 A_{1}+2 A_{2}, \\
16+16 A_{2}+\sum_{i=2}^{\left\lfloor\frac{n}{2}\right\rfloor} f(2 i) A_{2 i} \geq 16 \sum_{i=0}^{\left\lfloor\frac{n}{2}\right\rfloor} A_{2 i} \geq 8.2^{s},
\end{gathered}
$$

where we have used that fact that $f(2 i) \geq 16$ since $\frac{3 n+1}{4}$, the unique zero of $f(x)$, is an odd integer. Putting all these pieces together we obtain

$$
\begin{aligned}
& 2^{s}\langle f(x)\rangle=\sum_{i=0}^{n} f(i) A_{i} \\
\geq & f(0)+f(1) A_{1}+f(2) A_{2}+\sum_{i=2}^{\left\lfloor\frac{n}{2}\right\rfloor} f(2 i) A_{2 i} \\
\geq & f(0)-16+f(1) A_{1}+(f(2)-16) A_{2}+8.2^{s} \\
> & (3 n+5)\left(3 n-7+4 A_{1}+2 A_{2}\right)+8.2^{s} \\
= & (3 n+5)\langle f(x)-8\rangle+8.2^{s},
\end{aligned}
$$

in which the strict inequality comes from the $f(0)$ term. Taking into account of $\langle f(x)\rangle>8$ we obtain $2^{s}>3 n+5$, i.e., $n-k \geq 2 m+6$.

In the case of $a=1$, i.e., $n=N_{m}^{1}$ with $m \geq 0$ we define $g(x)=(3 n+2-4 x)(3 n-2-4 x)$ which is nonnegative on integers because $\frac{3 n+2}{4}$ is an integer. It is obvious that as long as $n \geq 5$ we have $g(i)>2(3 n+2)$ for $i=1,2$ and most importantly $g(0)>(3 n+2)(3 n-4)$. If there exists a stabilizer code $[[n, k, 3]]$ then Eqs. $17 \mathrm{a}$ 17c must hold, which leads to $\langle g(x)\rangle=3 n-4+2 A_{1}+2 A_{2}$. As a result we have

$$
\begin{aligned}
2^{s}\langle g(x)\rangle & \geq g(0)+g(1) A_{1}+g(2) A_{2} \\
& >(3 n+2)\left(3 n-4+2 A_{1}+2 A_{2}\right) \\
& =(3 n+2)\langle g(x)\rangle,
\end{aligned}
$$

in which the strict inequality sign is due to the $g(0)$ term. Since $\langle g(x)\rangle>0$ we have $2^{s}>3 n+2$, i.e., $n-k \geq 2 m+6$.

It should be noted that the optimal stabilizer codes of parameters as given in Eq. 2) exist and construct is already given by the stabilizers in Table I with the stabilizers acting on qubits $V_{0}$ or $V_{1}$ being replaced by 6 stabilizers of the pure optimal stabilizer codes $[[9,3,3]]$ or $[[10,4,3]]$.

The stabilizer-like structures of our codes simplify significantly the encoding and decoding procedures. Let us suppose we have already the encoding and decoding circuits for the codes $\mathbb{D}_{(0, a)}$ and for the Gottesman's codes. With some additional controlled-not gates in front of the encoding circuits of these individual codes we obtain the encodings of our codes. To decode we have only to check at first the first $2 m$ generators in Table I, from which we can be sure wether the errors happen on some U-block or not. If yes we use the decodings for Gottesmans codes and if not then we have only to decoding $\mathbb{D}_{(0, a)}$ with the detailed circuit in the case of $a=0$ being given in [12].

We acknowledge the financial support of NNSF of China (Grant No. 90303023, 10675107, and 10705025) and the A*STAR grant R-144-000-189-305.

Note added. On finishing the paper another infinite family of genuine nonadditive codes has been reported in [22].

[1] P. W. Shor, Phys. Rev. A, 2, 2493 (1995).

[2] C. H. Bennett, D. P. DiVincenzo, J. A. Smolin, andW. K.Wootters, Phys. Rev. A 54, 3824 (1996).

[3] A. Steane, Phys. Rev. Lett. 77, 793 (1996).

[4] E. Knill and R. Laflamme, Phys. Rev. A 55, 900 (1997).

[5] D. Gottesman, Phys. Rev. A 541862 (1996).

[6] D. Gottesman, arXive: quant-ph/9705052.

[7] A. Calderbank, E. Rains, P. Shor, and N. Sloane, Phys. Rev. Lett. 76, 405 (1997).

[8] A. Calderbank, E. Rains, P. Shor, and N. Sloane, IEEE Trans. Inform. Theory, 44, 1369 (1998).

[9] E.M. Rains, R. H. Hardin, P.W. Shor, and N.J.A. Sloane, Phys. Rev. Lett. 79, 953 (1997).

[10] E.M. Rains, IEEE Trans. Inf. Theory 45, 266 (1999).

[11] J.A. Smolin, G. Smith and S. Wehner, Phys. Rev. Lett. 99, 130505 (2007).

[12] S. Yu, Q. Chen, and C.H. Oh, arXiv: 0709.1780v1 [quant-ph]

[13] S. Yu, Q. Chen, C.H. Lai, and C.H. Oh, Phys. Rev. Lett. 101, 090501 (2008)

[14] M. Grassl and M. Roetteler, Proc. 2008 IEEE Int. Symp. on Inf. Theory (ISIT 2008), 300 (Toronto, Canada, July 2008).

[15] A. Cross, G. Smith, J. Smolin, and B. Zeng, IEEE Trans. Inf. Theory 55, 433 (2009).

[16] D. Gottesman, arXive: quant-ph/9607027

[17] E. Rains, IEEE Trans. Inform. Theory 44, 1388 (1998); ibid, IEEE Trans. Inform. Theory 45, 2361 (1999).

[18] P. Shor and R. Laflamme, Phys. Rev. Lett. 78, 1600 (1997).

[19] A. Ashikhmin and S. Litsyn, IEEE Trans. Inform. Theory 45, 1206 (1999).

[20] D. Schlingemann and R.F. Werner, Phys. Rev. A 65, 012308 (2001).

[21] M. Hein, J. Eisert, and H.J. Briegel, Phys. Rev. A 69. 062311(2004).

[22] M. Grassl, P. Shor, G. Smith, J. Smolin, and B. Zeng, arXiv: 0901.1319 [quant-ph]. 